\title{
BMJ Open Understanding rural caregivers' experiences of cancer care when accessing metropolitan cancer services: a qualitative study
}

\author{
Anna Ugalde, ${ }^{\oplus 1}$ Sarah Blaschke, ${ }^{2}$ Anna Boltong, ${ }^{3,4}$ Penelope Schofield, ${ }^{2,5,6}$ \\ Sanchia Aranda, ${ }^{7,8}$ Jo Phipps-Nelson, ${ }^{2,6}$ Suzanne K Chambers, ${ }^{9}$ \\ Meinir Krishnasamy, ${ }^{3,8,10}$ Patricia M Livingston ${ }^{1}$
}

To cite: Ugalde A, Blaschke S, Boltong A, et al. Understanding rural caregivers' experiences of cancer care when accessing metropolitan cancer services: a qualitative study. BMJ Open 2019;9:e028315. doi:10.1136/ bmjopen-2018-028315

- Prepublication history for this paper is available online. To view these files, please visit the journal online (http://dx.doi org/10.1136/bmjopen-2018028315).

Received 2 December 2018 Revised 5 May 2019 Accepted 29 May 2019

Check for updates

(C) Author(s) (or their employer(s)) 2019. Re-use permitted under CC BY-NC. No commercial re-use. See rights and permissions. Published by BMJ.

For numbered affiliations see end of article.

Correspondence to

Dr Anna Ugalde;

a.ugalde@deakin.edu.au

\section{ABSTRACT}

Objective To explore the experiences of cancer caregivers who live in rural Australia and travel to a metropolitan cancer health service to access cancer treatment.

Design A qualitative study using semistructured, audiorecorded interviews conducted between December 2017 and July 2018 with caregivers and social workers. Thematic analysis using interpretative descriptive techniques performed on textual interview data within a critical realist paradigm to develop understanding of rural caregivers' lived experiences.

Setting Participants were from rural areas attending a metropolitan cancer centre in Australia and social workers. Participants 21 caregivers (16 female) of people with cancer living in rural Australia within a minimum distance of $100 \mathrm{~km}$ from the metropolitan cancer centre where they access treatment, and five social workers employed at a metropolitan cancer service with experience of working with rural patients and caregivers.

Results Thematic analysis developed two overarching themes: theme 1: caregiving in the rural setting describes the unique circumstance in which caregiving for a person with cancer takes place in the rural setting at considerable distance from the cancer service where the person receives treatment. This is explored in three categories: 'Rural community and culture', 'Life adjustments' and 'Available supports'. Theme 2: accessing metropolitan cancer services captures the multiplicity of tasks and challenges involved in organising and coordinating the journey to access cancer treatment in a metropolitan hospital, which is presented in the following categories: 'Travel', 'Accommodation' and 'Health system navigation'.

Conclusions Caregivers who live in rural areas face significant challenges when confronting geographic isolation between their rural home environment and the metropolitan setting, where the patient accessed cancer treatment. There is a need for healthcare services to identify this group to develop feasible and sustainable ways to provide interventions that have the best chance of assisting rural caregivers in supporting the patient while maintaining their own health and well-being.
Strengths and limitations of this study

- This study collected two separate datasets using interviews with cancer caregivers and social workers in order to explore an under-researched topic from the caregiver perspective within a healthcare context.

- The methodology generated two themes that provide a rich understanding of the experience of cancer caregivers, with data collected from both caregivers and social workers.

- The study was informed by previous studies with rural and regional cancer patients, which provided insight into successful study procedures for this specific cohort including, for example, effective recruitment strategies and interview schedules.

\section{INTRODUCTION}

Informal caregivers represent a large and growing group who assume a pivotal role in providing care to people in need. ${ }^{12}$ In Australia, approximately one-third of caregivers are estimated to spend up to 40 hours or more per week in their caregiving role, accounting for an estimated 1.9 billion hours of unpaid care. ${ }^{1}$ Most people with cancer may have a partner, family member or close friend who, out of preference or necessity, assists them in meeting their care needs. ${ }^{34}$ While such a caregiving role can be rewarding and gratifying, ${ }^{5}$ supporting a person with cancer can present multiple challenges and result in the caregiver feeling burdened. ${ }^{6}$ Consequently, several negative impacts have been associated with taking on a caregiving role: reduced health-related quality of life, greater psychological distress, work productivity impairment, increased healthcare usage and stress-related comorbidities such as depression, anxiety and insomnia, ${ }^{78}$ and caregivers can 
suffer greater levels of distress and anxiety as compared with the patients they care for. ${ }^{9}$ Furthermore, caregivers report having various supportive care needs, particularly relating to disease-specific and health services information, and psychological and emotional support. ${ }^{9}$ It is known that their needs can persist over long periods of time and remain unmet even after the patient completes treatment. ${ }^{10}$ Thus, the prevailing perception that caregivers are more often persons providing support rather than needing support must be questioned, ${ }^{11}{ }^{12}$ and the task of adequately supporting caregivers must be addressed as a part of routine service provision.

Several factors can present additional complexity and challenge to caring for a person with cancer. It is well established that cancer outcomes in rural areas are poor relative to urban populations. ${ }^{13-15}$ Geographic isolation, greater distance to healthcare services, limited transportation options and variations in socioeconomic status and employment options all contribute to poorer rural cancer outcomes. ${ }^{13-16}$ Lack of information about available emotional, practical and financial support services and travel and accommodation assistance while away from home further contribute to the challenges facing rural people with cancer. ${ }^{17}$ Additionally, it is known that rural populations are more likely to experience socioeconomic disadvantage including lower levels of education and employment opportunities and reliance on government support. ${ }^{1}$ Access to radiation and chemotherapy services in rural Australia is improving; nevertheless, rural patients are often required to travel to metropolitan health services to access specific treatment. ${ }^{18}$ Given that timely and appropriate cancer treatment is associated with improved cancer outcomes, ${ }^{19}{ }^{20}$ and the fact that distance between home and cancer treatment services has been associated with lower rates of treatment ${ }^{21}{ }^{22}$ and poorer cancer outcomes, ${ }^{23}{ }^{24}$ it is important to understand how rural people deal with the specific barriers presented by their unique circumstance and how these may be overcome.

Studies have examined the experience and needs of people with cancer who live in rural areas, ${ }^{25}$ finding that they experience complex decision making, ${ }^{19}$ and they are required to consider socioeconomic factors related to travel and being away from home in order to access treatment. ${ }^{26}$ To date, however, the experience of rural caregivers who assist patients in accessing metropolitan cancer services has not been investigated, leaving a gap in understanding about their role in supporting and caring for rural people with cancer. Given the significance of caregiver contributions to patient care, ${ }^{2} 27$ and the possible negative impacts on their own health and well-being, ${ }^{1}{ }^{28-32}$ it is important to ensure mechanisms are in place to support caregivers. In the clinical setting, social workers provide various types of support such as psychosocial assessment, emotional support counselling, information provision and education, community referrals and assisting patients and families in navigating the health service and system. ${ }^{33}$ Their diverse clinical role enables them to address a broad array of issues encountered by patients and families.

The aim of this study was to understand rural caregivers' experiences of supporting patients in accessing cancer treatment in metropolitan settings from the perspectives of both cancer caregivers and oncology social workers.

\section{METHOD}

\section{Study design}

This research adopted a qualitative design using semistructured interviews to elicit lived experiences of rural Australians caring for a person with cancer who is required to access a metropolitan cancer service. A critical realist ${ }^{34}$ approach was adopted, which aligns with the study's phenomenological aim to investigate subjective first-person experience, and the pragmatic aim to inform health services development based on a better understanding of the challenges facing cancer caregivers who live in rural settings. Research procedures and reporting followed the Consolidated Criteria for Reporting Qualitative Research guidelines. ${ }^{35}$

\section{Participants and recruitment}

Two participant groups were recruited from a major Australian metropolitan health service. These included caregivers of patients with cancer who resided in an area requiring long-distance travel of more than $100 \mathrm{~km}$ to access cancer treatment or care at the metropolitan service. Social workers who were employed at the cancer service with experience working with rural caregivers were eligible to participate. Recruitment took place between December 2017 and July 2018, an 8-month period due to research assistant availability and pragmatic considerations. A purposive sampling strategy was applied to achieve a broad representation of experiences. ${ }^{36}$ Social workers with relevant work experience were identified by the head of department who has knowledge of team members' case loads including regular contact with patients and caregivers from rural areas. All social workers saw a minimum of 10 rural patients a month. Identified social workers were emailed study information and were invited to participate. Rural patients were identified through the in-hospital accommodation booking system and were emailed a study description and participation information sheet and invited to forward these to the person they identified as their caregiver.

\section{Public and patient involvement}

This research included participation by cancer caregivers who are the population under investigation. We did not directly involve patients, the public or caregivers in the development of this study.

\section{Data collection}

Face-to-face (in the hospital setting) or telephone one-off interviews were conducted by researchers trained in qualitative interviewing who did not have an clinical or 


\section{Box 1 Semistructured interview questions}

\section{Caregiver interview questions}

- How would you describe your role in assisting (patient name) with their cancer diagnosis or other health condition?

- Can you tell me about the travelling you need to do in the care for (patient name)?

- How were you involved in (patient name) decision to come to (name of metropolitan service) for treatment, if at all?

- In what ways do you feel travelling to (name of metropolitan service) has impacted you?

- How much information and support did you receive regarding your trip to (name of metropolitan service) for your treatment?

\section{Social worker interview questions}

- Based on your experiences, what are the different needs experienced by families and caregivers who travel to Peter Mac, compared with those who reside in metropolitan settings?

- What are the impacts of travelling from rural communities on caregivers?

- Do you feel rural and regional patients and caregivers receive enough information and support to come to Peter Mac for treatment?

otherwise established relationship with any of the participants (SB and a trained research assistant). Two separate datasets were generated: one using caregiver data and one using social worker data. A semistructured interview guide was designed based on successful examples from previous research with caregivers of cancer patients ${ }^{31}$ and caregivers of patients with motor neuron disease ${ }^{37}$ (see box 1). Interviews with social workers were guided by a set of questions developed to retrieve specific information related to their experience working with rural caregivers (see box 1). At the start of each interview, the researchers introduced themselves as affiliated with the institution facilitating the research and explained their role in assisting with the research project. Sociodemographic questionnaires were administered. The mean duration of interviews was $32 \mathrm{~min}$ (range 11-69 min). All interviews were audio-recorded and transcribed verbatim; field notes were not made. Participants were not provided a copy of the transcript for comment or correction. All researchers have relevant $\mathrm{PhD}$ degrees and were working in research or health service roles.

\section{Data analysis}

First, all data were sorted into predefined subject headings within each dataset (caregiver and social worker) as determined by the aim of the study, which was to explore the rural caregiver experience when travelling to a metropolitan cancer centre to access cancer treatment. Subject headings included, for example, 'Information and financial assistance', 'Easing the burden on rural people' and 'Navigating metropolitan services'. Next, thematic analysis $^{38}$ using interpretive descriptive techniques ${ }^{39}$ was performed by SB to explore emerging and recurring concepts within participants' responses. Textual data were inductively coded and interpreted in a constant comparative manner whereby salient concepts were labelled as codes. Codes were then logically grouped into larger categories. Interpretive analysis sought meaningful connections between categories within each dataset with the aim to understand and describe shared experiences of each participant group. Thematic description was further developed to fully capture the salience of participants' reported experiences. Initial analyses were conducted separately on each dataset with caregiver experience data being of primary interest. Social worker data were used to glean contextual understanding of caregivers' experiences in the metropolitan healthcare setting. Relevant connections were identified in the last step when overlaps and statements were identified to finalise the overarching themes. An inter-rater reliability process was undertaken to check interpretative congruity as is recommended to ensure rigour in qualitative analysis procedures and trustworthiness of findings. ${ }^{40}$ This included a second member of the research team (AU) reading the raw textual data and the developed coding structure. Incongruences in interpretations were discussed until agreement was reached. Descriptive statistics were used to summarise participants' sociodemographic information. Computing software was used to manage the data including NVivo version $10^{41}$ and Microsoft Excel.

\section{RESULTS}

One hundred and sixty-four emails were sent to patient email addresses to result in 21 caregivers ( $\mathrm{n}=16$ female; $76 \%$ ) providing signed, informed consent and participating in an interview. The number of emails received, read and opened are not known. Caregivers resided in three different states in Australia, and most (90.5\%) lived with the patient at the time of study recruitment. The study cohort included caregivers with varied types of relationship to the patient. Sociodemographic information for each participant is provided in table 1. Five female social workers with experience of working with rural patients and caregivers at a metropolitan cancer service participated. Their demographic and professional information are also presented in table 1 .

Analysis generated two overarching themes: 'Caregiving in the rural setting' and 'Accessing metropolitan cancer services'. The first overarching theme describes the unique circumstance in which a person assumes a caregiving role for a family member who is diagnosed with cancer and who wishes to access treatment in a metropolitan hospital. The second overarching theme captures the multiplicity of tasks and challenges involved in organising and coordinating the journey to access cancer treatment in a metropolitan hospital. Table 2 shows the supporting categories for each theme and provides illustrative quotes, with accompanying participant numbers. C01-C21 represent caregiver quotes and SW01-05 represent social worker quotes.

\section{Theme 1: caregiving in the rural setting}

Rural participants described their caregiving experiences against the context of their particular rural community. 
Table 1 Participant characteristics

\begin{tabular}{lr}
\hline Caregiver characteristics $\mathbf{( n = 2 1 )}$ & $\mathbf{n}(\%)$ \\
\hline Sex & \\
Female & $16(76)$ \\
Male & $5(24)$
\end{tabular}

Relationship to patient

\begin{tabular}{ll} 
Spouse/partner & $16(76)$ \\
\hline Son or daughter & $4(19)$ \\
\hline Parent & $1(5)$ \\
\hline Living situation & \\
\hline With the patient & $19(90)$ \\
\hline Not with the patient & $2(10)$ \\
\hline Tumour stream & \\
\hline Haematology & $6(29)$ \\
\hline Gastrointestinal & $5(24)$ \\
\hline Bone/soft tissue & $3(14)$ \\
\hline Melanoma/skin & $2(10)$ \\
\hline Other (breast, lung and & $3(14)$ \\
gynaecological) & \\
\hline Time spent as a caregiver & \\
\hline Less than a year & $5(24)$ \\
\hline 1-2 years & $7(33)$ \\
\hline 3-5years & $4(19)$ \\
\hline 6-10years & $3(14)$ \\
\hline 10+years & $2(10)$ \\
\hline & Mean (range) \\
\hline Caregiver age (years) & $59.95(32-83)$ \\
\hline Social worker characteristics (n=5) & $\mathbf{n}(\%)$ \\
\hline Sex & $5(100 \%)$ \\
\hline Female & Mean (range) \\
\hline & $45.80(30-60)$ \\
\hline Age & $13.60(6-21)$ \\
\hline Years worked as a social worker & $5.45(2-16)$ \\
\hline Years employed at health service & \\
\hline & \\
\hline
\end{tabular}

They discussed making several life adjustments as a consequence of a cancer diagnosis in the family and they reported on the support resources available to them to assist with their life situation. This overarching theme is represented by three categories: rural culture and community, life adjustments, and available supports.

\section{Rural culture and community}

Caregivers described mixed experiences regarding their local community and its role in caring for a family member with cancer, which diverged based on the level of perceived social support they were able to draw from their community. The majority of caregivers reported feeling embedded within a supportive community consisting of nearby family, friends or neighbours who were able to provide practical and emotional support. These participants discussed feeling a loss of community when having to spend time away from home to receive cancer treatment. Other caregivers, however, reported a lack of support due to social estrangement caused by geographic isolation, neighbours being of older age and therefore unable to provide support or an attitude of stoicism and self-sufficiency preventing either party from engaging in helping or help-seeking behaviour. Social workers reflected on their clinical encounters with rural families and described instances of struggle when families felt separated from their community support due to distance. One social worker discussed her observation of rural patients whose 'stoic' attitude created additional barriers for their caregiver to seek social support, and based thereon, noted that patient and caregiver social needs may differ.

\section{Life adjustments}

Caregivers discussed a range of life adjustments following a cancer diagnosis, which could impact on the entire family unit. Three caregivers reported making major life changes by relocating their home in order to gain better access to the metropolitan cancer service, reduce travel time, mitigate cost and gain closer proximity to family support.

The necessity for caregiver and patient to spend time away from home impacted on other family members too. Young and adolescent children were at the forefront of caregivers' concern when feeling responsible for the wellbeing of the family unit. Leaving children unattended and alone at home or requesting of them to take alternative transport to school caused additional worry. Caregivers and social workers discussed particularly challenging scenarios caused by unanticipated and abrupt changes to treatment plans, which demanded a fast decision to either leave the patient without caregiver support or for the caregiver not to return home as planned to care for the children.

Lack of practical home assistance was discussed in the context of neglecting ongoing domestic duties and farm work. Some caregivers had to accept a decline in living standard due to their extended absence and limited capacity to maintain the home and farm. Some caregivers discussed reluctance to hand over farm responsibilities to avoid imposing on others or simply because self-sufficiency and independence were preferred. To this end, one caregiver described the purchase of new work equipment to accommodate her husband's declining health condition and limited mobility. Other female caregivers reported having to take on new and physically vigorous farm tasks while their partner travelled unaccompanied to the city for cancer treatment. Of particular note was an observation made by C16 regarding the worry experienced by rural farmers during the bushfire season. In her view, some rural patients may need to decline treatment in a metropolitan hospital during this time. 


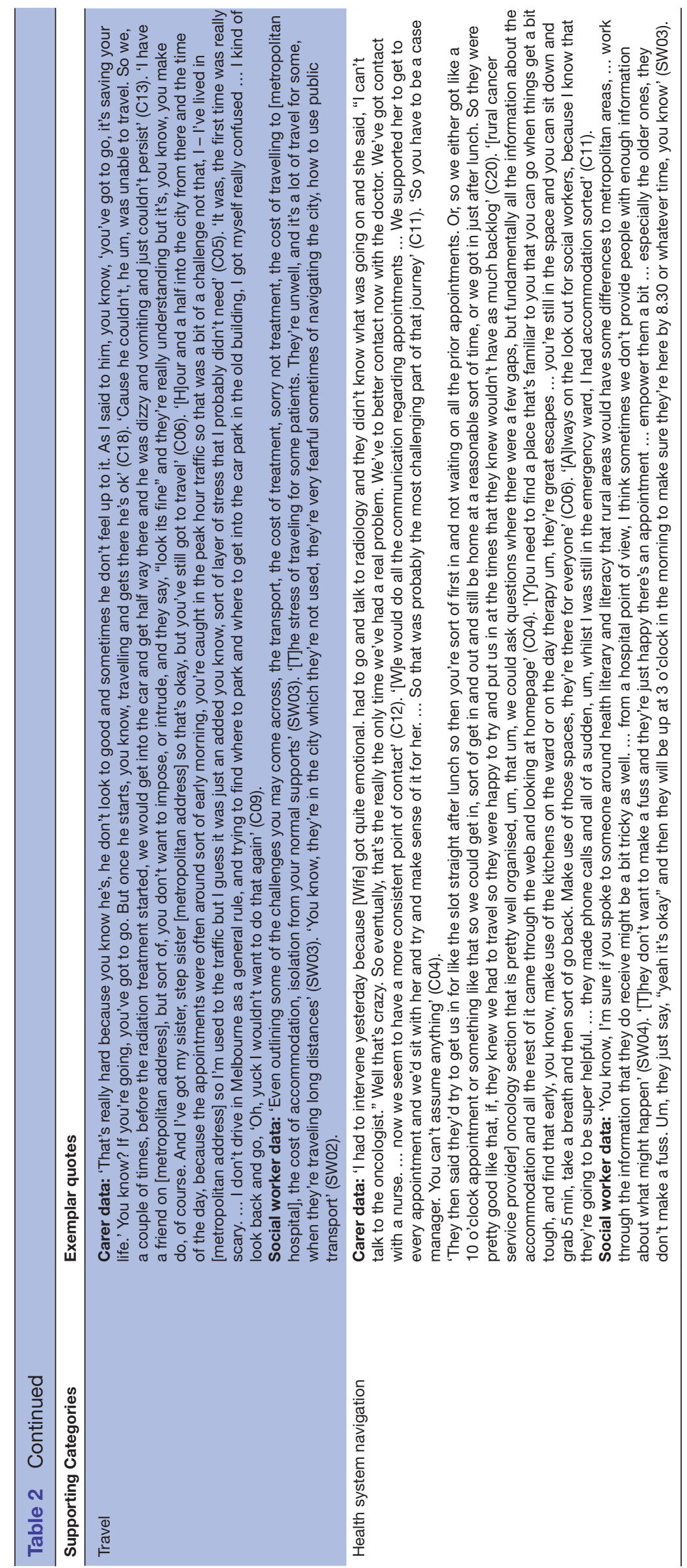


Loss of family income was discussed as a challenge and was attributed to patients' absence from work as well as caregivers' need to take leave from work to care for the patient. Similar adjustments and loss were faced by a student-aged caregiver who deferred her university education until both her parents had completed cancer treatment and she was relieved from frequently taking time away to drive and accompany her parents to the metropolitan hospital. While aware of the detriment caused by some life adjustments, the opportunity to access highquality cancer treatment was described, on the whole, to outweigh the burdens. Several caregivers accepted these burdens while dealing with cancer when living in a rural setting.

\section{Available supports}

Caregivers discussed the availability of resources to assist them in providing patient care at home. Among these were visits from the district nurse and assistance with acquiring and fitting amenities such as raised handrails and raised beds. Some caregivers discussed negative experiences related to assistance in the home, which was mainly caused by healthcare workers being perceived as intrusive. While personal questions asked by healthcare workers were accepted as being of clinical nature, rural caregivers, nonetheless, experienced a breach of privacy. One person reported that 'city people' are not able to fully understand and appreciate the needs and attitudes of rural people. In this regard, caregivers described their preference for privacy in social interactions and their appreciation of more secluded lifestyles. This was also reported by social workers who similarly felt that healthcare workers with rural backgrounds were better placed to support rural families dealing with cancer. Thus, caregivers as well as social workers identified the need for culturally sensitive support, with both groups indicating that support services for rural people require attention to the cultural aspects of their care needs.

\section{Theme 2: accessing metropolitan cancer services}

Rural caregivers assumed several essential tasks in regards to preparing and undertaking the journey from the rural setting to the city in order to access cancer treatment. They discussed their role in planning and coordinating three major components of the journey, which are presented below as the following three categories: travel, accommodation and health system navigation.

\section{Travel}

All caregivers discussed their decision-making process when considering travel to access treatment providers, and several factors contributed to choosing a metropolitan service over a local one. These included word of mouth or personal past experience or personal preference for a specific service provider or individual specialist, the perception that quality of treatment and care was higher in a specialised cancer facility, the convenience of gaining easy access to a range of specialist services within one location and preference for an integrated care approach focused on cancer treatment specifically. Finally, some caregivers perceived not having a choice and felt that treatment decisions were made by their doctor. Long-distance travel presented a complex planning process requiring the caregiver to carefully balance several factors. The patient's state of health and level of fitness was a major factor when considering the most appropriate mode of transport that would allow the patient to endure long-distance travel. Depending on the patient's physical condition, caregivers were required to organise multiple forms of private or public transport and assistance.

Early morning appointments were discussed by caregivers as particularly inconvenient, requiring an early start to avoid high-traffic times to arrive on time. Social workers recounted instances of elderly rural patients and caregivers travelling during the night in order to keep early morning appointments.

A further consideration was given to caregivers' own level of confidence with driving in the city. Participant C14, for example, described how her husband took on driving immediately after treatment and against doctor's advice due to her low level of confidence with driving in the city. Caregivers discussed their strategies for overcoming their fear of city driving. They described practising their driving skills during 'test drives' to the health service, which also provided familiarity with the route to the hospital and the parking amenities, though this was considerably time intensive. Some caregivers resorted to seeking travel assistance from family and friends or from health organisations offering patient transport. Caregivers with previous exposure to city driving noted great relief for having gained familiarity with the city environment before having to travel in this high stress situation.

\section{Accommodation}

Caregivers found it challenging to organise accommodation that was affordable yet in close proximity to the hospital. Social workers attested to this challenge and noted accommodation as being one of the most challenging aspects for rural families when attending the metropolitan cancer service. Caregivers and social workers held similar views about the importance of early provision of information about travel and accommodation options, which helped to reduce worry and uncertainty. Knowledgeable caregivers reported several avenues through which they accessed information about available supports and accommodation options. Some conducted their own internet research to retrieve information relevant to their circumstance. Other caregivers reported receiving information through their social networks and word of mouth or through a central contact person in the metropolitan service such as a nurse coordinator or social worker. In-hospital accommodation was preferred for convenience and for cost saving given the availability of government subsidies for this type of accommodation. However, in-hospital facilities were not always available 
to caregivers due to insufficient supply, or other hospital requirements under which caregivers were required to seek external and self-funded accommodation.

Accommodation cost presented a significant financial dilemma for some rural families who had to cover out-of-pocket expenses with their own life savings. Social workers reported working with rural families who struggled to meet these financial demands. Specific challenges were mentioned regarding the availability of information about financial supports such as government reimbursement. Some caregivers found out about financial aid options later on and sometimes too late to reclaim costs that were incurred in previous years. Other caregivers discussed their difficulty as relating to the demanding and lengthy administrative processes involved in making reimbursement claims. Once claims were lodged, several rural families struggled to self-finance costs until the reimbursement was received, which were reported to take up to several months.

In this context, social workers advised of the usefulness of screening rural patients for unmet needs and advocated for mechanisms that could allow early detection of support needs to prevent later escalation of issues and reduce the resources needed to manage these.

\section{Health system navigation}

Caregivers assumed several responsibilities once they arrived at the metropolitan cancer service. They provided various forms of personal assistance to the patient and assisted in navigating the health service and the physical hospital environment. Social workers were frequently accessed for assistance with these navigation tasks. In-hospital volunteer services were mentioned by caregivers and social workers as helpful. Caregivers' positive experiences of the volunteer service were underpinned by feeling recognised as 'country' people who may require additional practical and navigation assistance in the hospital setting. This caring attitude caused rural families to perceive volunteers as particularly personable and attentive towards their needs.

When presenting for multiple appointments during a single visit, caregivers described their tasks in navigating and locating different services in the hospital, checking in for appointments and assisting the patient during appointments. Their role included conveying information and supporting patients in comprehending and retaining clinical communication. When unable to attend and assist the patient themselves, efforts were made to organise another person to assist the patient during appointments. For some this presented discomfort when having to recruit support from their city networks or request a member of their local community to travel to the city.

Caregivers assumed varying levels of responsibility for future appointment booking and care coordination. Some caregivers discussed positive experiences and described the service as considerate of their specific needs and flexible in regards to appointment booking to meet their travel requirements. However, caregivers and social workers described mixed approaches and differing levels of self-advocacy about families' care needs. Caregivers who felt that they could not rely on service staff to communicate clinical information across different services assumed a more proactive role in following up with hospital administration. They took it on themselves to ensure that appointment times aligned with their travel and accommodation needs. In contrast, social workers described rural families, particularly older age patients and caregivers, as people who avoided 'making a fuss'. As a consequence, they were noted to accept unnecessary inconveniences.

Social workers also discussed the importance of empowering rural families to speak up about their needs and the necessity to implement support systems that ensure visibility and advocacy of rural families and their unique needs.

\section{DISCUSSION}

The present study provides new insights into the specific scenarios encountered by rural caregivers who assist patients in travelling to a metropolitan hospital in order to access cancer treatment. The findings extend previous research on the experiences and challenges facing rural people who deal with cancer ${ }^{19}{ }^{26}$ and address an area of supportive care need that, to date, has received little attention.

There are several key findings from this study. The results demonstrate that there is extensive planning, impact and burden associated with travel for family caregivers. As per similar studies that focus on the patient, results suggest that decision making in regards to travel, and the actions required to execute this decision, are complex and need to balance multiple factors. ${ }^{19}$ Several socioeconomic impacts were associated with accessing cancer treatment away from home. Notably, the burden of travelling and related financial cost emerged as particularly challenging for many rural families, which reflects findings from previous research with rural people who deal with cancer. ${ }^{26}$

We propose that interventions, supports or programmes to assist families and caregivers who travel for cancer treatment need to be appropriate and delivered within the context of rural community. The results support implementation of mechanisms in the oncology setting that increase the visibility of rural families, and identification of this group is likely to be an important first step. As advocated by social work participants in this study, early screening of support needs, ideally when first presenting at the metropolitan service, could prevent later escalation of issues, which may require more resource-intensive responses.

It is noteworthy that not all rural caregivers welcomed assistance and some described having very particular preferences for the type of support that they were willing to accept. This is consistent with previous research that identified people from rural and regional areas as preferring 
a self-sufficient lifestyle, having stoic attitudes and being less likely to ask for help. ${ }^{42}$ In our study, limited understanding and low sensitivity towards cultural differences between rural and urban populations were associated with negative experience. High importance was given to information and support resources that are specific and relevant to the needs of rural people. Both caregivers and social workers considered the relevance of support workers' own backgrounds and life experience with preference for health workers who come from rural background themselves. Similar to previous research focused on people with cancer, there may be specific groups of rural caregivers who most benefit from support, such as those experiencing financial burden ${ }^{26}{ }^{43}$ and those who have young children at home. ${ }^{44}$ Support for these groups, including understanding and linking in with available community resources, could be a focus of future research. Development of policies to ensure mechanisms for isolated patient travel reimbursement is also warranted.

Limitations of this study include generalisability of findings. The study was solely conducted at one metropolitan cancer service. It cannot be concluded that the range of experiences is exhaustive when considering cancer experiences in other rural settings to attend other metropolitan hospitals for treatment. Additionally, there are known caregiver-patient relationships not represented in our study cohort. For example, non-kin rural caregivers did not participate in this study who may face different or additional challenges. Given these limitations, we concur with other researchers who suggest the need for prospective studies on people from rural settings affected by cancer. ${ }^{42}$ Finally, differences between caregivers who choose to participate in research and those who choose not to participate need to be considered. Our recruitment strategy did not allow for reasons for non-participation to be well understood. It is therefore likely that a host of issues encountered by rural caregivers remain unreported and notably absent from the research literature.

In conclusion, our study identified the existence of a range of distinct challenges facing rural caregivers who assist patients in accessing metropolitan cancer services. These related to geographical distance as well as practical and, in particular, financial factors associated with travel and time spent away from home. While participants reflected on their circumstance with humility and great willingness to assume additional burdens, they were clear about the challenging nature of their responsibilities as a rural person caring for a family member with cancer.

\footnotetext{
Author affiliations

${ }^{1}$ School of Nursing and Midwifery, Faculty of Health, Deakin University, Geelong, Victoria, Australia

${ }^{2}$ Department of Cancer Experiences Research, Peter MacCallum Cancer Centre, Parkville, Victoria, Australia

${ }^{3}$ Victorian Comprehensive Cancer Centre, Parkville, Victoria, Australia

${ }^{4}$ Melbourne School of Population and Global Health, The University of Melbourne, Parkville, Victoria, Australia

${ }^{5}$ School of Psychological Sciences and Iverson Health Innovation Research Institute, Swinburne University, Hawthorn, Victoria, Australia
}

${ }^{6}$ Sir Peter MacCallum Department of Oncology, The University of Melbourne, Parkville, Victoria, Australia

${ }^{7}$ Cancer Council Australia, Sydney, New South Wales, Australia

${ }^{8}$ Department of Nursing, The University of Melbourne, Parkville, Victoria, Australia

${ }^{9}$ University of Technology Sydney, New South Wales, Australia

${ }^{10}$ Centre for Cancer Research, University of Melbourne, Parkville, Victoria, Australia

Acknowledgements We would like to thank Erin Tutty for research assistant support for this study.

Contributors All authors conceived the study and were responsible for design of the study; $A U$ and $S B$ drafted the protocol with input from the team. The draft was reviewed and commented on by AB, PS, SA, JP-N, SKC, MK and PL. SB assisted with data acquisition with support from AU and JP-N, and SB and AU conducted data analyses. All authors assisted with interpretation and contributed to drafting and critically revising the manuscript and related study materials. Correspondence across the team discussed any study issues, findings and interpretation of results. All authors read and approved the final manuscript.

Funding Anna Ugalde was the recipient of a Victorian Cancer Agency Early Career Seed Grant.

Competing interests None declared.

Patient consent for publication Not required.

Ethics approval Ethical approval was obtained from the Peter MacCallum Cancer Centre Human Research Ethics Committee (Reference number LNR/17/PMCC/189) and Deakin University (2017-368).

Provenance and peer review Not commissioned; externally peer reviewed.

Data sharing statement Please contact the corresponding author in regards to any data sharing requests. Please note there are ethical restrictions on sharing of data.

Open access This is an open access article distributed in accordance with the Creative Commons Attribution Non Commercial (CC BY-NC 4.0) license, which permits others to distribute, remix, adapt, build upon this work non-commercially, and license their derivative works on different terms, provided the original work is properly cited, appropriate credit is given, any changes made indicated, and the use is non-commercial. See: http://creativecommons.org/licenses/by-nc/4.0/.

\section{REFERENCES}

1. Australian Institute of Health and Welfare. Australia's welfare 2017: in brief. 2017. Australia's welfare series numbe 1. AUS 214. Canberra: AlHW, 2017.

2. National Centre for Social and Economic Modelling. Who's going to care? Informal care and an ageing population: Report prepared for Carers Australia. Canberra: University of Canberra, 2005.

3. Ugalde A. Development of an instrument to measure self-efficacy in informal caregivers of people with advanced cancer, in School of Health Sciences. Melbourne: The University of Melbourne, 2012.

4. Currow DC, Christou T, Smith J, et al. Do terminally ill people who live alone miss out on home oxygen treatment? An hypothesis generating study. J Palliat Med 2008;11:1015-22.

5. Grant G, Ramcharan P, McGrath M, et al. Rewards and gratifications among family caregivers: towards a refined model of caring and coping. J Intellect Disabil Res 1998;42:58-71.

6. Papastavrou E, Charalambous A, Tsangari H. Exploring the other side of cancer care: the informal caregiver. Eur J Oncol Nurs 2009;13:128-36.

7. Goren A, Gilloteau I, Lees M, et al. Quantifying the burden of informal caregiving for patients with cancer in Europe. Support Care Cancer 2014;22:1637-46.

8. Burnette D, Duci V, Dhembo E. Psychological distress, social support, and quality of life among cancer caregivers in Albania. Psychooncology 2017;26:779-86.

9. Sklenarova $\mathrm{H}$, Krümpelmann $\mathrm{A}$, Haun MW, et al. When do we need to care about the caregiver? Supportive care needs, anxiety, and depression among informal caregivers of patients with cancer and cancer survivors. Cancer 2015;121:1513-9.

10. Girgis A, Lambert SD, McElduff $P$, et al. Some things change, some things stay the same: a longitudinal analysis of cancer caregivers' unmet supportive care needs. Psychooncology 2013;22:1557-64.

11. Harding R, Higginson IJ. What is the best way to help caregivers in cancer and palliative care? A systematic literature review of interventions and their effectiveness. Palliat Med 2003;17:63-74. 
12. Richardson A, Plant H, Moore S, et al. Developing supportive care for family members of people with lung cancer: a feasibility study. Support Care Cancer 2007;15:1259-69.

13. Baade PD, Youlden DR, Coory MD, et al. Urban-rural differences in prostate cancer outcomes in Australia: what has changed? Med $J$ Aust 2011;194:293-6.

14. Satasivam P, O'Neill S, Sivarajah G, et al. The dilemma of distance: patients with kidney cancer from regional Australia present at a more advanced stage. BJU Int 2014;113 Suppl 2:57-63.

15. Roder D, Zorbas H, Kollias J, et al. Risk factors for poorer breast cancer outcomes in residents of remote areas of Australia. Asian Pac J Cancer Prev 2013;14:547-52.

16. Australian Institute of Health and Welfare. A snapshot of men's health in regional and remote Australia. Canberra: AlHW, 2010.

17. Davis C, Williams $P$, Redman S, et al. Assessing the practical and psychosocial needs of rural women with early breast cancer in Australia. Soc Work Health Care 2003;36:25-36.

18. Fox $P$, Boyce $A$. Cancer health inequality persists in regional and remote Australia. Med J Aust 2014;201:445-6.

19. Bergin R, Emery J, Bollard R, et al. How rural and urban patients in Australia with colorectal or breast cancer experience choice of treatment provider: A qualitative study. Eur J Cancer Care 2017;26:e12646.

20. Neal RD, Tharmanathan P, France B, et al. Is increased time to diagnosis and treatment in symptomatic cancer associated with poorer outcomes? Systematic review. $\mathrm{Br} J$ Cancer 2015;112:S92-107.

21. Sundaresan P, King M, Stockler M, et al. Barriers to radiotherapy utilization: consumer perceptions of issues influencing radiotherapyrelated decisions. Asia Pac J Clin Oncol 2017;13:e489-e496.

22. Waran E. Radiotherapy: The tyranny of distance. Aust J Rural Health 2017;25:64-5.

23. Ruseckaite R, Sampurno F, Millar J, et al. Diagnostic and treatment factors associated with poor survival from prostate cancer are differentially distributed between regional and metropolitan Victoria, Australia. BMC Urol 2016;16:54.

24. Celaya MO, Rees JR, Gibson JJ, et al. Travel distance and season of diagnosis affect treatment choices for women with early-stage breast cancer in a predominantly rural population (United States). Cancer Causes Control 2006;17:851-6.

25. Haigh MM, Baxi S, Lyford M, et al. Cancer support services: Are they meeting the needs of rural radiotherapy patients? Eur J Cancer Care 2019;28:e12904.

26. Hegney D, Pearce S, Rogers-Clark C, et al. Close, but still too far. The experience of Australian people with cancer commuting from a regional to a capital city for radiotherapy treatment. Eur J Cancer Care 2005;14:75-82.

27. Yabroff KR, Kim Y. Time costs associated with informal caregiving for cancer survivors. Cancer 2009;115:4362-73.
28. Weitzner MA, McMillan SC, Jacobsen PB. Family caregiver quality of life: differences between curative and palliative cancer treatment settings. J Pain Symptom Manage 1999;17:418-28.

29. Given CW, Given B, Stommel M, et al. The caregiver reaction assessment (CRA) for caregivers to persons with chronic physical and mental impairments. Res Nurs Health 1992;15:271-83.

30. Grov EK, Dahl AA, Moum T, et al. Anxiety, depression, and quality of life in caregivers of patients with cancer in late palliative phase. Ann Oncol 2005;16:1185-91.

31. Ugalde A, Krishnasamy M, Schofield P. Role recognition and changes to self-identity in family caregivers of people with advanced cancer: a qualitative study. Support Care Cancer 2012;20:1175-81.

32. Rhee YS, Yun YH, Park S, et al. Depression in family caregivers of cancer patients: the feeling of burden as a predictor of depression. $J$ Clin Oncol 2008;26:5890-5.

33. Pockett R, Dzidowska M, Hobbs K. Social work intervention research with adult cancer patients: a literature review and reflection on knowledge-building for practice. Soc Work Health Care 2015;54:582-614.

34. Maxwell JA. A realist approach for qualitative research. US: SAGE Publications Inc, 2012.

35. Tong A, Sainsbury P, Craig J. Consolidated criteria for reporting qualitative research (COREQ): a 32-item checklist for interviews and focus groups. Int J Qual Health Care 2007;19:349-57.

36. Onwuegbuzie AJ, Leech NL. Sampling designs in qualitative research: making the sampling process more public. Qual Report 2007;12:238-54.

37. Anderson NH, Gluyas C, Mathers S, et al. "A monster that lives in our lives": experiences of caregivers of people with motor neuron disease and identifying avenues for support. BMJ Support Palliat Care 2016:bmjspcare-2015-001057.

38. Corbin J, Strauss A. Basics of Qualitative Research: Procedures and Techniques for Developing Grounded Theory. US: SAGE Publications Inc, 2008

39. Thorne S, Kirkham SR, O'Flynn-Magee K. The analytic challenge in interpretive description. Int J Qual Methods 2004;3:1-11.

40. Strauss A, Corbin JM. Basics of qualitative research: Grounded theory procedures and techniques. US: SAGE Publications Inc, 1990.

41. QSR. NVivo qualitative data analysis software. Version 10: QSR International Pty Ltd, 2014.

42. Butow PN, Phillips F, Schweder J, et al. Psychosocial well-being and supportive care needs of cancer patients living in urban and rural/regional areas: a systematic review. Support Care Cancer 2012;20:1-22.

43. Fenn KM, Evans SB, McCorkle R, et al. Impact of financial burden of cancer on survivors' quality of life. J Oncol Pract 2014;10:332-8.

44. Semple CJ, McCance T. Parents' experience of cancer who have young children: a literature review. Cancer Nurs 2010;33:110-8. 